\title{
Evolución, vulnerabilidad e impactos económicos y sociales de El Niño 2015-2016 en América Latina
}

\author{
Evolution, vulnerability and the economic and social impacts \\ of El Niño 2015-2016 in Latin America
}

\author{
Rodney Martínez ${ }^{1}$ \\ Eduardo Zambrano ${ }^{2}$ \\ Juan José Nieto ${ }^{3}$ \\ Julián Hernández $z^{4}$ \\ Felipe Costa ${ }^{5}$
}

\section{Resumen}

El presente artículo documenta en detalle la evolución de las variables oceánicas y atmosféricas más relevantes durante el evento cálido El Niño 2015-16, así como sus manifestaciones sobre América Latina en lo relacionado con precipitaciones, temperatura y eventos extremos. El Niño 2015-16 fue, junto a los de 1997-1998 y 1982-1983, uno de los más fuertes desde 1950. Afectó a millones de personas, principalmente en América Latina. Durante el 2015-16, se experimentó una combinación inédita de El Niño, la fase cálida de la Oscilación Decadal del Pacífico y el período más cálido del planeta, desde que existen registros. Se realizó una recopilación de fuentes oficiales y reportes de prensa para establecer los impactos sociales y económicos más significativos en América Latina, con énfasis en el sector agrícola y la seguridad alimentaria. Aunque se evidencia una amplia gama de impactos en los países, los más significativos de El Niño 2015-16 se reflejaron principalmente en el agravamiento de las sequías en México, Centroamérica, El Caribe y al norte de Sudamérica, con eventos puntuales de precipitaciones extremas y las consecuentes inundaciones en varios países, desde el centro hasta el sur del continente.

Palabras clave: El Niño; vulnerabilidad; América Latina; gestión de riesgos; impactos socioeconómicos.

\begin{abstract}
1 Centro Internacional para la Investigación del Fenómeno de El Niño, Ecuador. r.martinez@ciifen.org

2 Centro Internacional para la Investigación del Fenómeno de El Niño, Ecuador. e.zambrano@ciifen.org

3 Centro Internacional para la Investigación del Fenómeno de El Niño, Ecuador. j.nieto@ciifen.org

4 Centro Internacional para la Investigación del Fenómeno de El Niño, Ecuador. j.hernandez@ciifen.org

5 Centro Internacional para la Investigación del Fenómeno de El Niño, Ecuador. f.costa@ciifen.org
\end{abstract}

This article documents in detail the evolution of the most relevant oceanic and atmospheric variables during the warm phase of El Niño 2015-16, as well as its manifestations on Latin America in relation to precipitation, temperature and extreme events. El Niño 2015-16 is comparable to those of 1997-1998 and 1982-1983, and it has been one of the most powerful since 1950. It affected millions of people, mainly in Latin America. During 2015-16 the global climate experienced an unprecedented combination of El Niño, the Pacific Decadal Oscillation and the hottest period on the planet, since records began. Official sources and press reports were compiled to pinpoint the most consequential social and economic impact on Latin America, in particular with regard to the agricultural sector and food security. Although it had a 
wide range of repercussions in the countries, the most significant effects of El Niño 2015-16 were mainly associated with the exacerbated drought in the region, the extreme precipitation events and the subsequent flooding.

Keywords: El Niño; vulnerability; Latin America; risk management; socioeconomic impacts.

\section{Introducción}

El evento El Niño/Oscilación del Sur (ENOS), o simplemente "El Niño", se refiere a un evento climático de escala global en el que se produce un calentamiento anómalo de buena parte del Pacífico Tropical que motiva a su vez un debilitamiento o colapso de los vientos alisios. Ambas condiciones, se realimentan mutuamente en lo que se conoce acoplamiento océano-atmósfera permitiendo la evolución del evento por varios meses. Esta perturbación se propaga a través de la atmósfera y afecta el clima de buena parte de las regiones del mundo, convirtiéndose en el factor más influyente en la variabilidad climática natural.

El evento El Niño 2015-16 fue declarado por la Organización Meteorológica Mundial [OMM] como uno de los tres más fuertes registrados desde 1950, junto con los de 1982-83 y 1997-98 (OMM, 2016). Las características de este evento generaron impactos significativos alrededor del globo, principalmente sobre la agricultura y seguridad alimentaria. Se estima que más de 60 millones de personas fueron afectadas por las consecuencias ligadas a sequías, inundaciones y eventos extremos de calor y frío. Se requirieron cerca de USD 4,000 millones para afrontar la inseguridad alimentaria generada tras la pérdida de entre 50 y 100\% de las cosechas solamente en Centroamérica. Entre las afectaciones de este evento también se atribuyen desplazamientos forzados de la población, luego de los colapsos de medios de vida y daños a la infraestructura básica (Food and Agriculture Organization of the United Nations [FAO], 2016a; United Nations Office for the Coordination of Humanitarian Affairs [OCHA], 2016; United Nations International Strategy for Disaster Reduction [UNISDR], 2016).

Estas afectaciones además de poner en evidencia ciertos avances en gestión de riesgo en Latinoamérica, también han revelado los problemas existentes en cuanto al ordenamiento y manejo territorial, así como el flujo de información para la toma de decisiones. Se han puesto en evidencia los vacíos existentes para lograr la resiliencia climática. El Niño mostró una vez más que puede seguir sorprendiendo al mundo y afirmó la necesidad de profundizar aún más la comprensión de la vulnerabilidad de las poblaciones, sus ecosistemas y los medios de vida para poder gestionar los riesgos basados en evidencia. En buena parte de América Latina, las acciones son totalmente orientadas a responder a los impactos una vez producidos con pocos procesos verdaderos de prevención a corto y mediano plazo. Algunas de las posibles limitantes se asocian a un flujo limitado de información, poca confianza en las predicciones pero por sobre todo una incipiente gestión del territorio para reducir la exposición y un modelo de desarrollo en el que todavía no se inserta de forma efectiva los riesgos asociados a la variabilidad y el cambio climático.

En este artículo, el término impactos se emplea para describir los efectos negativos sobre los sistemas humanos y productivos generados por episodios meteorológicos y climáticos extremos derivados de El Niño 2015-16. Se hace énfasis en los impactos sobre el sector agrícola al ser generalmente el más afectado en la región, y del cual se obtuvo mayor información disponible.

\section{Metodología}

\subsection{Evolución de El Niño 2015-2016}

El año de 2015 fue de mucha actividad de las componentes zonales (sentido este-oeste) de los vientos ecuatoriales, seguidos de una serie de ondas Kelvin de gran magnitud que transportaron gran cantidad de energía calórica desde el borde occidental hasta el oriental del Pacífico. Este transporte hizo que la temperatura bajo la superficie de gran parte del Pacífico ecuatorial oriental presentara grandes regiones más cálidas de lo normal, lo cual constituye una de las particularidades que caracteriza la fase inicial de desarrollo del evento El Niño.

El primer evento intenso de los vientos del oeste con anomalías de hasta $16 \mathrm{~m} / \mathrm{s}$ que ocurrió en marzo (figura 1b), hizo que la primera onda Kelvin se desplazara por el Pacífico y llegara a la costa de América del Sur a fines de abril, provocando un calentamiento de más de $3^{\circ} \mathrm{C}$ de la temperatura superficial del mar (TSM) (figura la). Este transporte de calor permitió que la capa subsuperficial del Océano Pacífico central ya en marzo evidenciara una región cálida con más de $4^{\circ} \mathrm{C}$ sobre lo normal (figura 1c). 
Figura 1. (a) Anomalía del contenido de calor en la capa superior del océano Pacífico ecuatorial para el año de 2015. (b) Anomalía de la componente zonal de los vientos (m/s) en la capa de $850 \mathrm{hPa}$ sobre el Pacífico ecuatorial para el año de 2015. (c) Anomalía de la temperatura subsuperficial en el Pacífico ecuatorial para los meses de marzo, junio, septiembre y diciembre de 2015.
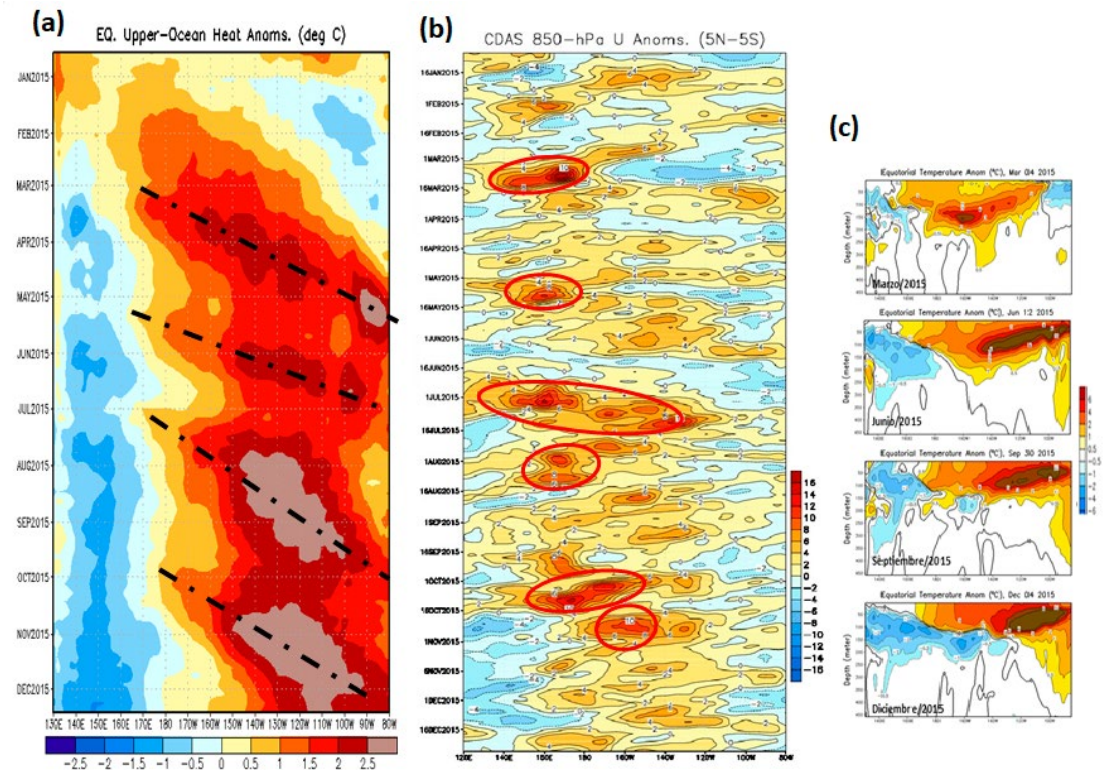

Fuente: Center (2009).

Figura 2. Anomalía de la Temperatura Superficial del Mar (TSM) en grados Celsius para noviembre y diciembre de 2015 y de enero 2016.
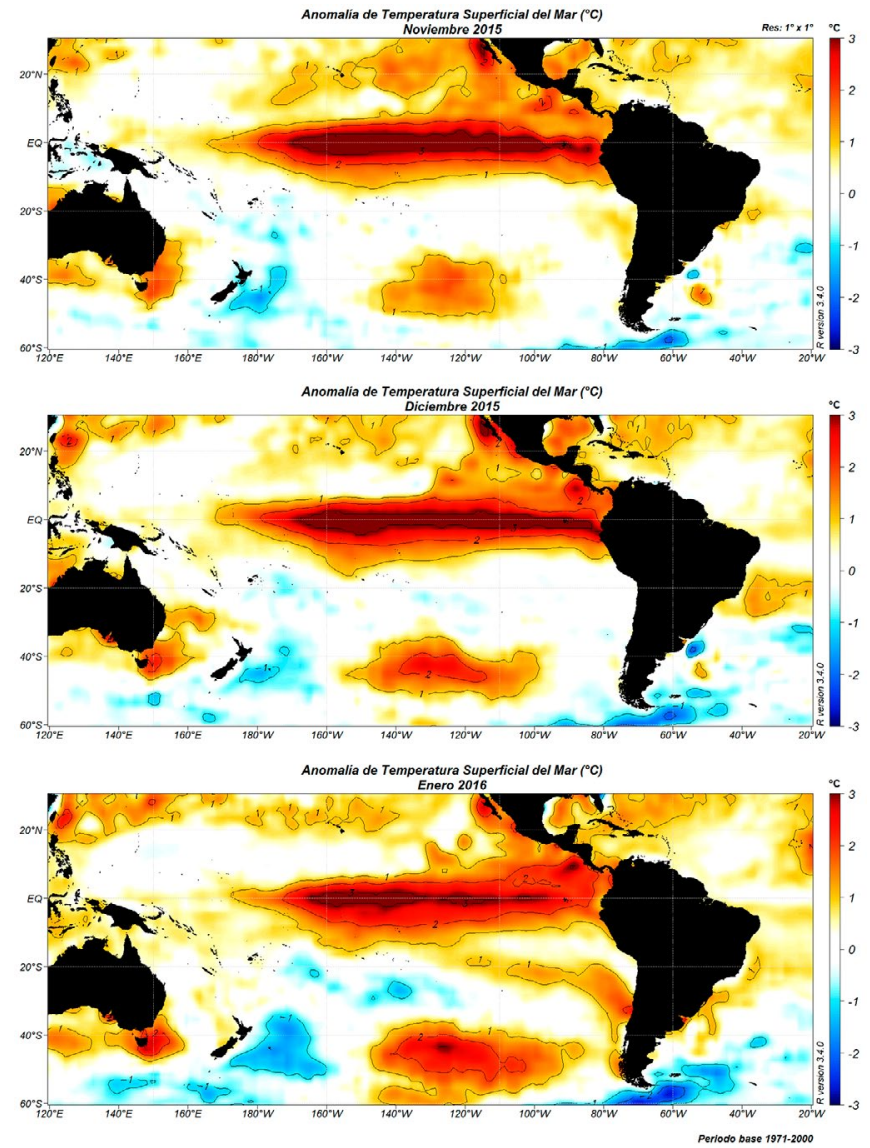

Fuente: Reynolds, Rayner, Smith, Stokes, \& Wang (2002). Elaboración propia. 
A partir de este calentamiento, se produjeron más ondas Kelvin que continuaron transportando calor al Pacífico Central y Oriental durante el resto del 2015, haciendo que el evento El Niño alcanzara una intensidad fuerte. Luego del primer pulso de vientos anómalos del oeste y el subsecuente desplazamiento de la primera onda Kelvin en marzo, durante el año, se presentaron más de cinco pulsos de viento del oeste con anomalías superiores a $10 \mathrm{~m} / \mathrm{s}$, distribuidos en mayo, julio, agosto, octubre y noviembre (figura 1b). Estos pulsos de viento, contribuyeron con el desarrollo de al menos tres ondas Kelvin que provocaron anomalías de más de $3^{\circ} \mathrm{C}$ en la TSM, siendo una en la primera quincena de mayo, otra a comienzos de julio y la última en octubre (figura la). Esto produjo anomalías positivas de la TSM de hasta $6^{\circ} \mathrm{C}$ en el este del Pacífico para el segundo semestre del año, mientras que en el oeste se produjo una anomalía negativa que llegó a $-2^{\circ} \mathrm{C}$ (figura 1c).

En abril del 2015 las anomalías positivas de la TSM se expandieron a toda la región del Pacifico Ecuatorial y gran parte del borde oriental del Pacífico Sur, alcanzando hasta $3^{\circ} \mathrm{C}$ al oeste de las Islas Galápagos $\left(95^{\circ} \mathrm{W}\right)$. En julio, las anomalías positivas de la TSM se habían consolidado en todo el Pacífico, evidenciando una distribución asimétrica con anomalías más cálidas en el hemisferio norte que en el hemisferio sur (figura 2).

Estas anomalías positivas se vieron, además, reflejadas por la profundización del calentamiento del océano Pacífico junto a la costa de Sudamérica. Del lado occidental del Pacífico ecuatorial se observó una anomalía negativa del agua subsuperficial (figura 1 y 2 ).

Todas estas características anómalas del sistema océano-atmosfera indicaron que la fase cálida de El Niño para noviembre-diciembre de 2015 y enero de 2016, había alcanzado su máxima intensidad y que, debido a su magnitud, ya era considerado uno de los eventos más fuertes de los últimos 50 años, junto a los eventos de los años 1982-83 y 1997-98.

Las fuertes anomalías tanto en los vientos de superficie como en los de la troposfera sobre la región ecuatorial generaron perturbaciones que se propagaron a manera de ondas de Rossby, generando teleconexiones en buena parte del continente americano, Asia, Oceanía y África. En Latinoamérica, desde mayo de 2015 hasta julio de 2016 aproximadamente, se produjo déficit de precipitaciones en la región de El Caribe, Centroamérica, gran parte de Venezuela, Colombia y Brasil. Por otro lado, se registró un exceso de precipitaciones en el noreste de Argentina, sur de Brasil, Paraguay y Uruguay (noviembre-diciembre de 2015), Ecuador y norte de Perú (noviembre de 2015-mayo de 2016) (figura 3).

Figura 3. Anomalía de precipitación (mm) para noviembre y diciembre de 2015 y enero de 2016.

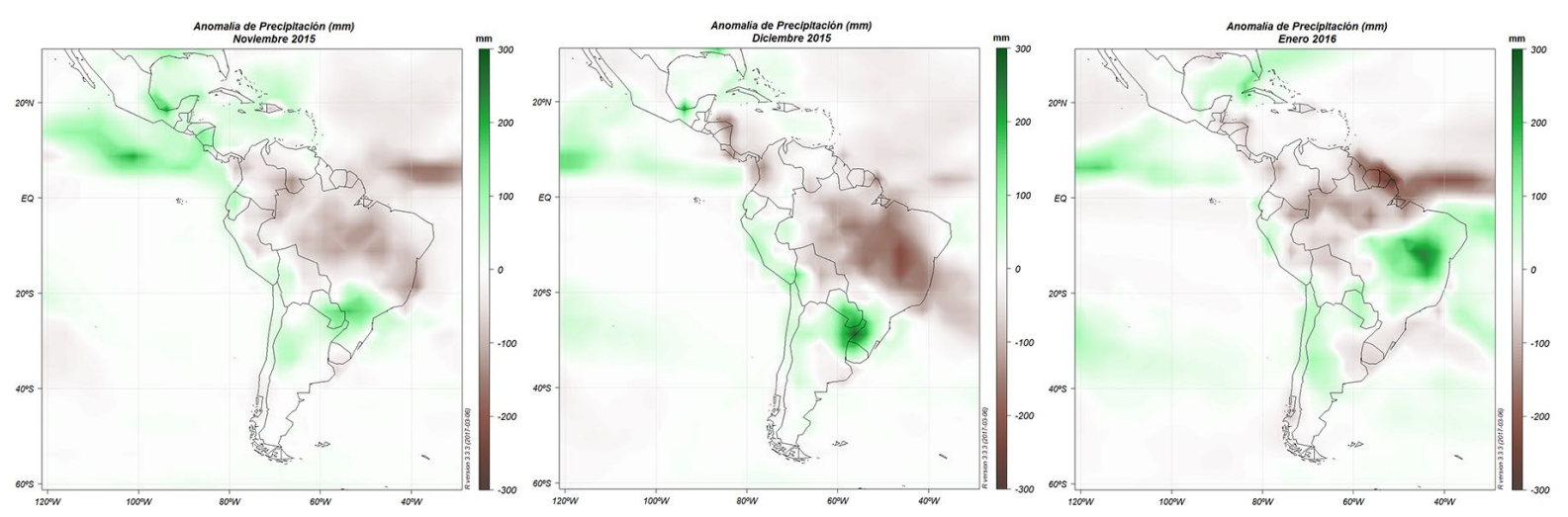

Fuente: Janowiak \& Xie (1999). Elaboración propia.

\subsection{Decaimiento de El Niño 2015-2016}

Durante los meses de diciembre y enero, las anomalías de temperatura superficial del mar en el pacífico Central-Oriental mantuvieron los valores más altos mientras los vientos del oeste, mantenían igualmente sus anomalías. A partir del mes de marzo del 2016, inició la fase de declinación de El Niño con una reducción sostenida de las anomalías de los vientos del oeste y la persistente actividad de las ondas Kelvin finalmente se detuvo por lo que no hubo aportes de calor en el Pacífico Tropical Oriental. Como consecuencia, las anomalías térmicas empezaron un descenso gradual a partir del mes de abril (figuras 4 y 5 ). 
Figura 4. (a) Anomalía del contenido de calor $\left({ }^{\circ} \mathrm{C}\right)$ en la capa superior del océano Pacífico ecuatorial para el período de enero a agosto de 2016. (b) Anomalía de la componente zonal de los vientos (m/s) en la capa de $850 \mathrm{hPa}$ sobre el Pacífico ecuatorial para el primer semestre de 2016. (c) Anomalía de la temperatura subsuperficial $\left({ }^{\circ} \mathrm{C}\right)$ en el Pacífico ecuatorial para febrero, marzo, abril y junio de 2016.

(a) EQ. Upper-Oceon Heot Anoms. (deg C)

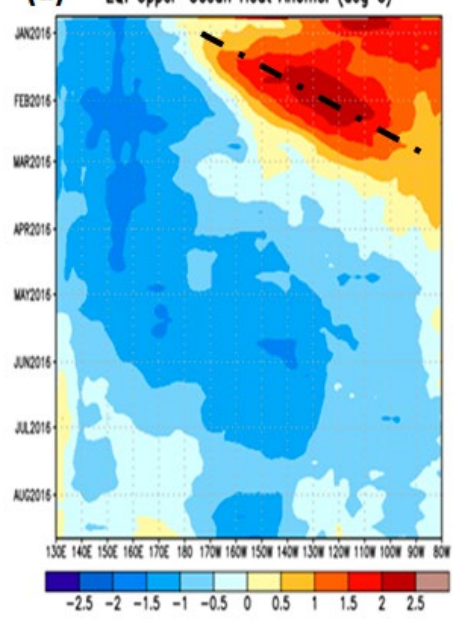

(b)

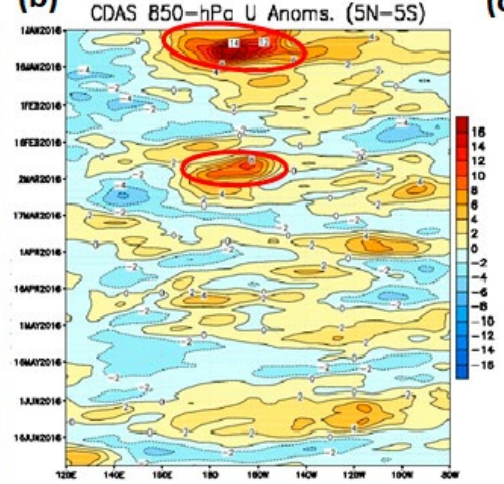

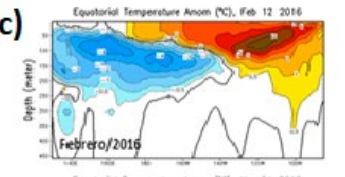
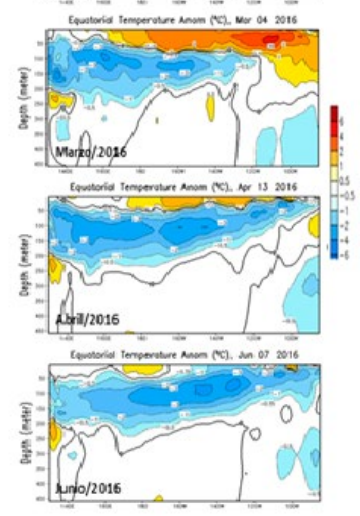

Fuente: Center (2009)

Figura 5. Anomalía de la Temperatura Superficial del Mar (TSM) en grados Celsius para febrero, abril y junio de 2016.
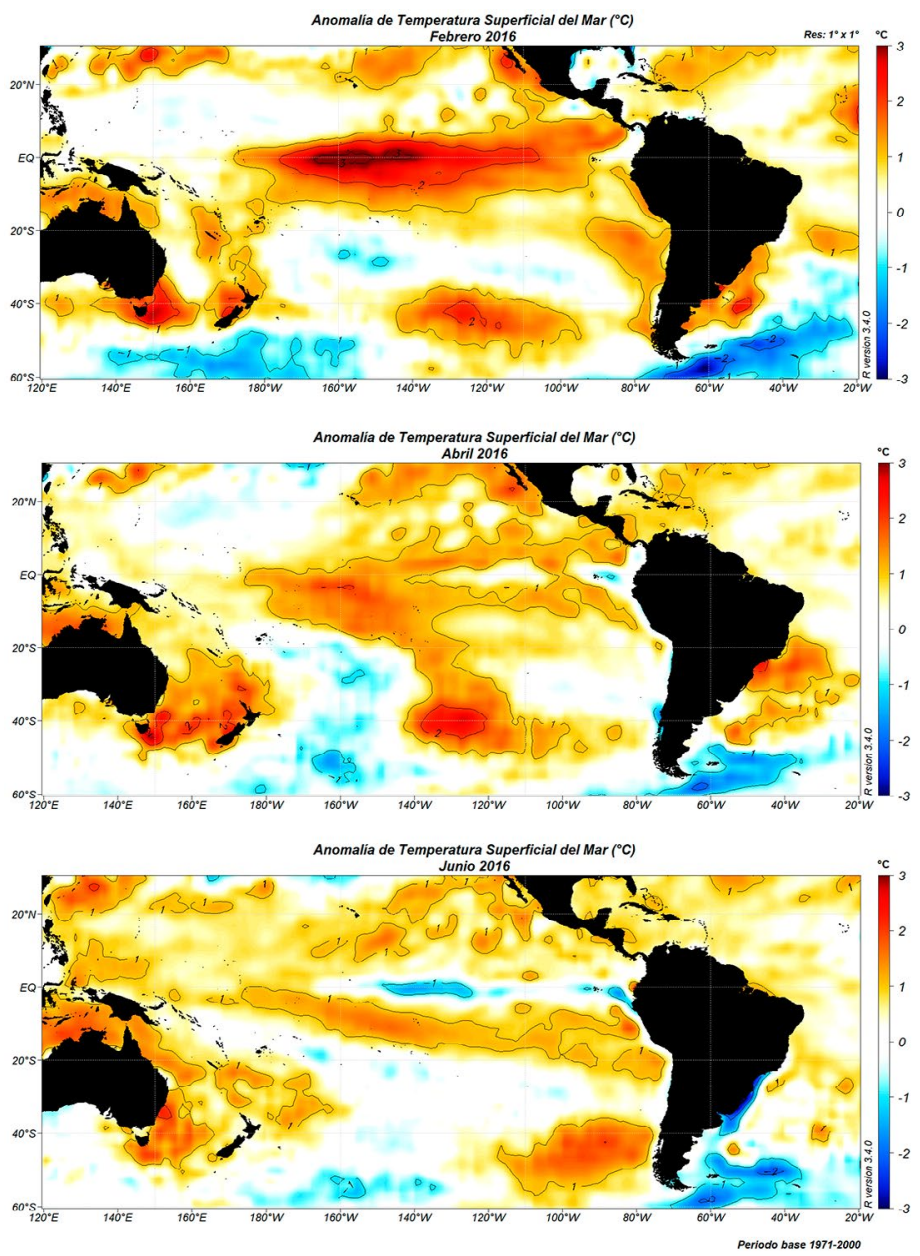

Fuente: Reynolds et al., (2002). Elaboración propia. 
Entre febrero y marzo, a nivel subsuperficial, en el Pacífico occidental se inició también la propagación hacia el este de masas de agua con temperatura de hasta $-3^{\circ} \mathrm{C}$ bajo lo normal (entre 10 y $40 \mathrm{~m}$ de profundidad). El rápido enfriamiento subsuperficial prosiguió durante las semanas subsiguientes, alcanzando en abril las costas de Sudamérica, quedando como remanente del fuerte calentamiento subsuperficial de los meses anteriores [20 meses aproximadamente] una delgada capa de agua cálida, ubicada muy cerca de la superficie. En junio, la capa de agua subsuperficial levemente fría se encontraba a lo largo de todo el Pacífico ecuatorial, señalando de esta manera el inicio de la fase neutra del ENSO en el Pacífico tropical (figura 4c).

Durante los primeros meses del 2016 continuó el déficit de precipitaciones en la región del El Caribe, Colombia, Venezuela y región noroccidental y central de Brasil. Sin embargo, en abril ya se apreciaron cambios significativos, presentándose valores sobre lo normal en Colombia y Venezuela, norte de Argentina, Uruguay y sur de Brasil. La región de Centroamérica continuó con valores deficitarios durante casi todo el primer semestre (figura 6).

La temperatura del aire fue superior a lo normal hasta el primer trimestre del 2016, excepto en el suroeste de Colombia, noreste de Brasil y Centroamérica. A partir de abril se observó un cambio significativo en la temperatura del aire en Sudamérica. Se registraron valores sobre lo normal en el sur donde se presentaron valores de hasta $-3^{\circ} \mathrm{C}$ por debajo del promedio [anomalías negativas]. Esta condición se acentuó en los meses posteriores, así, durante junio del 2016 en gran parte del centro de Sudamérica también se presentaron anomalías negativas. Para el resto de Sudamérica se observó ligeras anomalías positivas, especialmente en el noreste de Ecuador, oeste de Bolivia y centro de Brasil (figura 7).

Figura 6. Anomalía de precipitación (mm) para febrero, abril y junio de 2016.

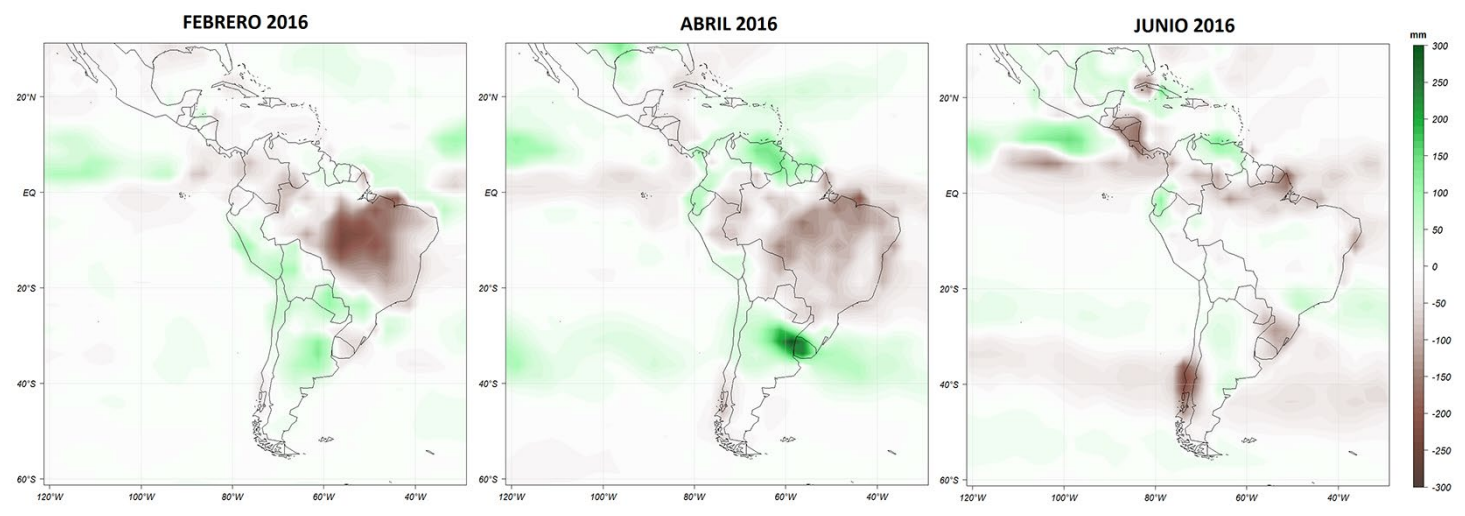

Fuente: Janowiak \& Xie (1999). Elaboración propia.

Figura 7. Anomalía de Temperatura del Aire $\left({ }^{\circ} \mathrm{C}\right)$ para febrero, abril y junio de 2016.

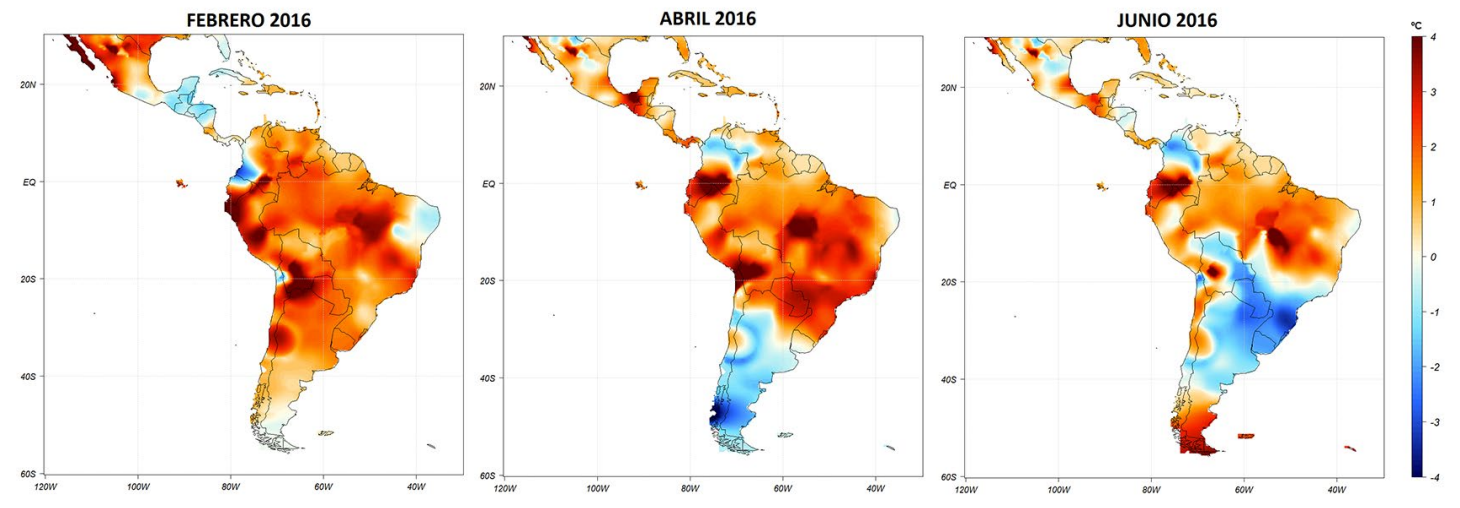

Fuente: Fan \& Van den Dool (2008). Elaboración propia

\subsection{Comparación con los eventos El Niño 1982-83 y 1997-98}

La distribución de la anomalía de la TSM para noviembre durante los eventos El Niño 1982, 1997 y 2015 en el Pacífico ecuatorial presentó una distribución simétrica entre dichos años. Para el último even- 
to, hubo un mayor calentamiento general el Pacífico norte. Sin embargo, en el Pacífico centro-oriental ecuatorial se destacó la similitud en la magnitud de las anomalías de la TSM y la extensión que ocuparon en el océano (figura 8). A nivel subsuperficial, la anomalía de la temperatura del mar presentó un comportamiento bastante similar entre los eventos 1997-98 y 2015-16, aunque en El Niño 2015-16 las anomalías cálidas fueron más superficiales y más extendidas a lo largo del ecuador. También se observó un ingreso más discreto de agua fría desde el oeste comparado con El Niño 1997-98 (figura 9).

Figura 8. Anomalía de la Temperatura Superficial del Mar $\left({ }^{\circ} \mathrm{C}\right)$ para el mes de noviembre de los años de 1982, 1997 y 2015.
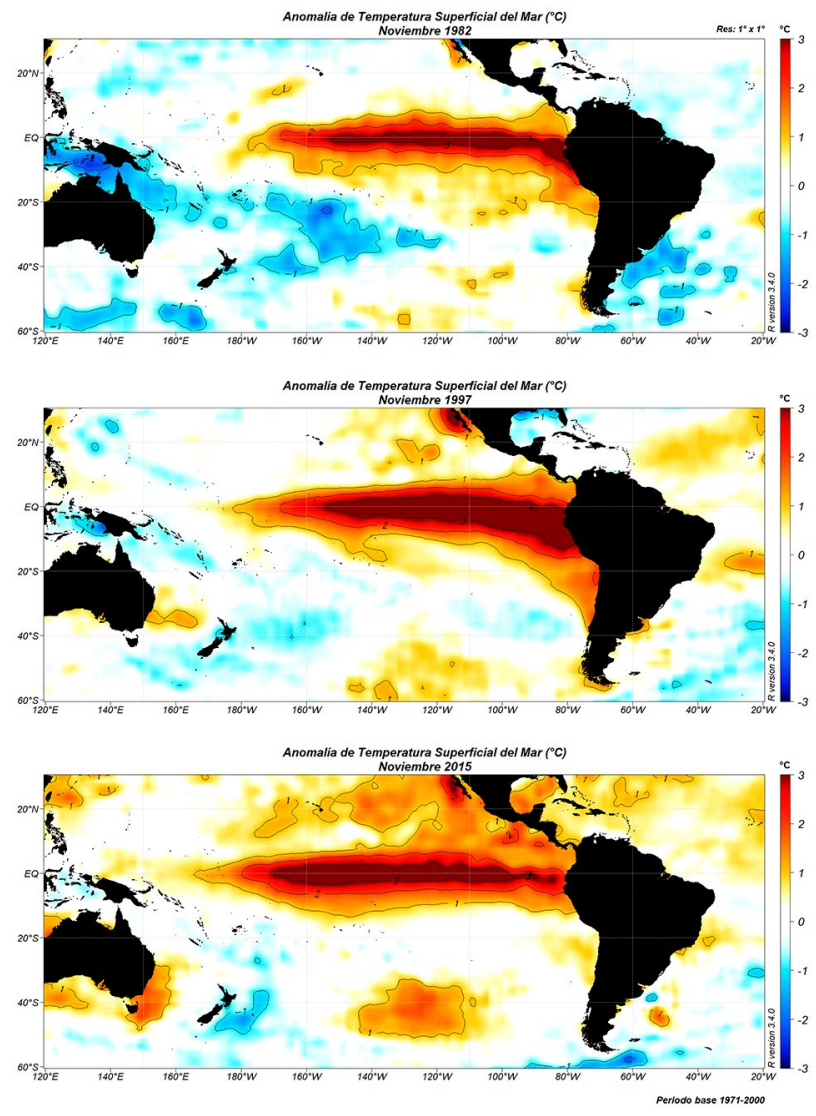

Fuente: Reynolds et al., (2002). Elaboración propia.

Figura 9. Anomalía de la temperatura subsuperficial del mar de octubre a enero para los años 1997/98 (izquierda) y 2015/16 (derecha).

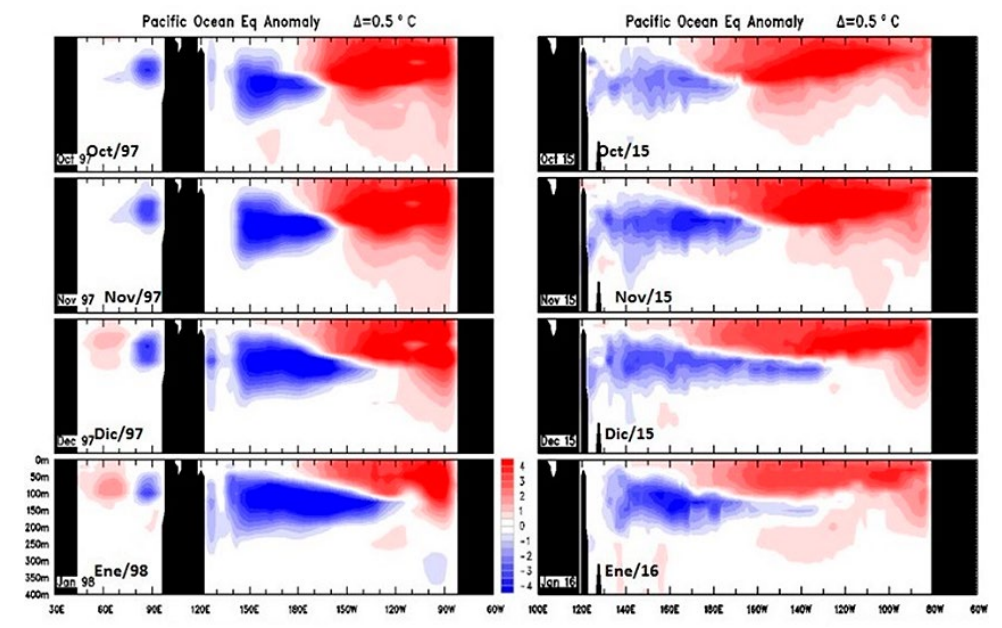

Fuente: Izquierda: BOM (1997). Derecha: BOM (2015). 


\section{Resultados}

Se realizó una compilación de los principales impactos económicos y sociales del evento El Niño 2015-16, para lo cual se ha dividido el análisis en tres sub-regiones: México y Centro América, El Caribe y Sudamérica. En los impactos económicos, se hace énfasis en el sector agrícola porque fue de este sector de dónde se pudo obtener datos con prontitud, lo que no ocurrió con los demás sectores. En los impactos sociales se puso énfasis en las implicaciones sobre la seguridad alimentaria. A pesar que durante el evento El Niño 2015-16, pudo hacerse un seguimiento de la evolución del evento y la información generada fue mucho mayor que en los eventos anteriores comparables, como los de 1982-83 y 1997-98, aun se evidencian impactos significativos en varias regiones de América Latina, posiblemente atribuibles a una deficiente prevención y flujo de información.

\subsection{Impactos en México y Centroamérica}

\subsubsection{Impactos económicos}

Centroamérica fue una de las regiones más azotadas por el evento El Niño 2015-16, particularmente los países que forman parte del Corredor Seco de Centroamérica como El Salvador, Guatemala, Honduras y Nicaragua. En estos países más de 3.5 millones de personas fueron directamente afectadas por la inseguridad alimentaria y necesitaron asistencia humanitaria después de la pérdida de cultivos de cereales, principalmente del maíz, y de granos como el frijol, que son considerados básicos para la seguridad alimentaria de la región. Para esta intensa y prolongada sequía, se estimó que más de 33\% de la población agrícola, perdió entre 50\% y el 90\% de sus cultivos, causando una brecha financiera de USD 17 millones (FAO, 2016d).

En El Salvador, para estos dos cultivos, maíz y fréjol, se estimaron pérdidas de más del 50\% durante el primer semestre de 2015, lo que representó alrededor de unos USD 28 millones (FAO, 2015). Mientras que Honduras presentó pérdidas de cerca de $60 \%$ del maíz y $80 \%$ de la producción de frijoles. Las consecuencias de estas pérdidas resultaron en el incremento de importaciones para evitar el desabastecimiento del mercado lo que dio lugar a un aumento de los precios de los alimentos en un 20\% (FAO, 2016b).

En las áreas más afectadas de Guatemala se calcularon pérdidas de entre el 50 y 100\% de las cosechas, afectando a más de 175 mil familias, con un volumen de pérdidas de más de USD 133 millones tan solo entre maíz y frijol negro. Escenario parecido se dio en Nicaragua, donde el 50\% del área total sembrada sufrió daños, mientras que en las zonas más afectadas, las pérdidas fueron totales. En estos dos países también se urgió tomar medidas de importación de productos básicos para garantizar la disponibilidad de alimentos y la estabilidad de sus precios ${ }^{6}$ (FAO, 2015).

En Costa Rica, tan solo en el primer semestre de 2015, las ventas del sector agrícola al exterior cayeron un $4.5 \%$ lo que provocó que se perdieran unos USD 250 millones en exportaciones. De eso, unos USD 200 millones estuvieron relacionados solamente con la piña y el banano (FAO, 2016a).

El sector agrícola en México sufrió los efectos de la presencia del huracán Patricia en el océano Pacífico, en octubre de 2015. Las afectaciones se dieron en los cultivos de cereales, aguacate y agave, materia prima para producir el tequila [lo que representó más del $80 \%$ de las pérdidas económicas totales en los centros productivos de este país]. Además, este huracán causó daños materiales, viales, en diversas infraestructuras y en zonas industriales, como Guadalajara, Aguascalientes y Monterrey. Todas las ciudades del occidente mexicano sufrieron pérdidas de alrededor de USD 10,000 millones según la Comisión Económica para América Latina y el Caribe [CEPAL $]^{7}$ (Univisión, 2015).

\subsubsection{Impactos sociales}

El segmento de población más vulnerable en los países de la región, lo constituyen familias de pequeños jornaleros y agricultores de subsistencia, que se caracterizan por tener bajos ingresos y ser muy dependientes del clima, puesto que trabajan en zonas agropecuarias que no disponen de riego controlado. Además, tienen limitado acceso a los servicios básicos de salud y educación y poseen dificultades para obtener la canasta básica (OCHA, 2015).

6 Guatemala es el único país en el corredor seco que ha logrado que los precios bajen respecto a agosto del año pasado gracias a las
importaciones de México y mayores suministros de la cosecha principal de 2015 en curso. 7 Estimaciones de Sergio Saldaña, consultor de desastres naturales del CEPAL. 
Los niveles de desnutrición aguda moderada y severa fueron evaluados por la Red de Sistemas de Alerta Temprana para la Hambruna [FEWS NET, por su sigla en inglés] en abril del 2015. Los resultados obtenidos indicaron un nivel de Desnutrición Aguda Moderada [DAM] del 0.8\% en la región occidental del corredor seco y 2.8\% en la región oriental de Centroamérica. En el caso de la Desnutrición Aguda Severa [DAS], se reportó un $0.8 \%$ y $0.3 \%$, respectivamente (OCHA, 2015).

En la búsqueda de mejores condiciones, se observó una alta tasa de emigración rural hacia centros urbanos y también hacia otros países, según un estudio de la Organización Internacional para las Migraciones [OIM] y el Programa Mundial de Alimentos [WFP, por su sigla en inglés] (OCHA, 2015). Además, se identificó por ejemplo, que al menos un miembro del $12 \%$ de las familias afectadas en Guatemala emigró, lo que representó alrededor de 25 mil a 30 mil personas que migraron por la inseguridad alimentaria, derivada de la hambruna provocada por la sequía (WFP, 2016a).

Con la afectación de más de 4.2 millones de personas, se declaró emergencia alimentaria. Tan solo en Guatemala se estimó que 1.5 millones de personas requirieron asistencia humanitaria tras el evento, con 720 mil personas en condiciones de inseguridad alimentaria severa, aproximadamente, el 10\% de la población del país. En Honduras, también por causa de la inseguridad alimentaria, se requirió asistencia humanitaria para el 15\% de la población del país (FAO, 2016b).

WFP y FAO catalogaron al este de El Salvador, Honduras, Guatemala y noroeste de Nicaragua como las zonas más afectadas y con alta prioridad de actuación para erradicar la inseguridad alimentaria (WFP, 2016a; FAO, 2016d).

\subsection{Impactos en El Caribe}

\subsubsection{Impactos económicos}

Las alertas por sequía fueron divulgadas por el Instituto de Meteorología e Hidrología de El Caribe [CIMH] para distintos países de la región, incluidos Antigua y Barbuda, Barbados, Dominica, Norte de Guyana, St. Kitts y Nevis, Santa Lucía, San Vicente y Las Granadinas, Trinidad y Tobago, norte de Surinam, Granada y Haití. Para estos países, los impactos se dieron principalmente en el sector agropecuario, influenciando, sobre todo, las exportaciones (WFP, 2016a).

Se estima que en la región caribeña hubo una reducción general de la actividad pesquera de aproximadamente el 25\% del volumen de pesca, según la Organización del Sector Pesquero del Istmo Centroamericano [SICA] (El Universo, 2016).

En Haití, la sequía severa causó pérdidas significativas en la actividad agraria, particularmente en las cosechas de las que dependen como insumo para el sustento básico familiar. Se reportaron pérdidas en la producción agrícola del $89 \%$, un $37 \%$ de desempleados en los hogares y un $61 \%$ de las familias vieron muy afectados sus recursos económicos. En el país se necesitaron al menos, USD 7.9 millones tras el paso del evento El Niño 2015-2016 (FAO, 2016a; WFP, 2016b).

\subsubsection{Impactos sociales}

En Haití, país más afectado de El Caribe, se estimó un total de 3.6 millones de habitantes en condiciones de inseguridad alimentaria. Esta cifra fue alcanzada tras la intensificación de la sequía en la región como resultado del evento El Niño 2015-16.

Por esto, la diversidad de la dieta sufrió una baja moderada en un 49\% de los hogares y severa en 31\%. La población disminuyó el consumo de alimentos ricos en proteínas, hierro y vitamina A, incrementando los problemas de desnutrición. A pesar de ello, los gastos con alimentación representaron $65 \%$ de los gastos totales del hogar para la mitad de la población. Estos datos son consecuencia directa de la sequía en un país donde el $80 \%$ de las familias no tienen reservas de alimento para el consumo doméstico (FAO ,2016b; WFP, 2016a; WFP, 2016b).

\subsection{Impactos en Sudamérica}

\subsubsection{Impactos económicos}

En América del Sur, los impactos climáticos de El Niño 2015-16 generaron daños a infraestructuras, reducción de la producción agropecuaria y afectaciones a la matriz energética y productiva. Todos de alguna forma resultaron en pérdidas económicas. 
En Ecuador, las pérdidas económicas del sector agropecuario se estimaron en USD 3.5 millones con más de 2 mil productores entre medianos y pequeños, afectados. Tanto en la región Costa como en la Sierra, se observó la disminución de la producción de papa, como resultado de las altas temperaturas y exceso de humedad. Hubo un total de $34.7 \mathrm{~km}$ de vía afectadas en todo el país por el exceso de precipitaciones, incidiendo en las comunicaciones, transporte y comercio (Secretaria de Gestión de Riesgos [SGR], 2016).

En Colombia una de las principales consecuencias de El Niño 2015-16, fue la sequía. Los principales cultivos afectados fueron los cultivos de exportación como el café, la papa y el arroz. De acuerdo a una estimación de la Federación Nacional de Cafeteros, El Niño, en 2015 causó daños a cerca de 90000 ha del cultivo, lo que representaba el $18 \%$ del total de cultivo de café en todo el país (RCN Radio, 2016). Según la agremiación, El Niño incidió en los costos de control de plagas. Al menos 50000 ha de papa se perdieron, lo que puso en riesgo la inversión de 200 millones de dólares de este cultivo. Otros cultivos afectados por la sequía fueron, arroz, caña de azúcar, palma aceitera, algodón y cacao, que sumarian un total de 2 millones de hectáreas.

En Venezuela, el efecto principal fue la sequía que afectó al país por un largo período y se vio acentuada por la presencia de El Niño 2015-16. Los mayores impactos se dieron sobre el sector hidroeléctrico, agrícola y ganadero. Los principales embalses del país vieron su cota de agua reducida a niveles graves que podían producir un colapso eléctrico en el país. De este modo, la producción de alimentos y el sistema agrícola en general también se vieron severamente afectados, reduciendo su capacidad de cultivo y distribución y consecuentemente un impacto directo en la seguridad alimentaria y la crisis económica por la que atraviesa el país.

En Perú, considerado el mayor abastecedor mundial de harina de pescado, vieron reducida su producción, las zonas más importantes de actividad pesquera y acuícola. Esta actividad es considerada uno de los motores de la economía peruana. Los impactos más intensos se dieron en Piura [norte] e Ica [centro-sur], tanto para la pesca artesanal, cuanto industrial. En la región de Ancash, la pesca industrial fue la más afectada, provocando una reducción en la producción de harina de pescado. En Puno [sureste], se registraron impactos en las actividades acuícolas (El Comercio, 2016a).

Las pérdidas económicas en Perú alcanzaron cifras aproximadas de USD 1100 millones, según la Asociación Peruana de Empresas de Seguros [APESEG]. Por otro lado, los daños en infraestructuras y comunicaciones evidencian el enorme impacto producido por El Niño en Perú (Gestión, 2015).

El sector agrícola de Bolivia sufrió pérdidas de al menos 31 mil hectáreas de cultivo y casi 16 mil cabezas de ganado desde noviembre de 2015. Además, se registraron daños materiales y afectaciones en infraestructuras, vías y en comunicaciones. El Estado dedicó USD 21.5 millones a partir del Plan de Emergencia Nacional por el Fenómeno de El Niño (FAO, 2016b).

En Brasil, El Niño 2015-2016 produjo sequías en la región noroeste, mientras que en la región sur se registraron excesos de precipitaciones. Debido a esto, los productores de arroz en el sur del país, responsables del 70\% de la producción nacional, sufrieron importantes pérdidas en la producción. El maíz tuvo una reducción de $14 \%$ en la cosecha, llegando a la producción más baja de los últimos cinco años. Los costos de la energía aumentaron del $4 \%$ al $8 \%$ en el 2016. En total se estiman pérdidas de alrededor de USD 300 millones en el sector agropecuario, con una reducción del 1.6\% en la producción de granos en 2015-16 incrementándose además, las importaciones de granos en el país (FAO, 2016c).

\subsubsection{Impactos sociales}

En Ecuador, según el balance de la Secretaría de Gestión de Riesgos, hasta el 30 de mayo de 2016, identificó unas 27 mil personas directamente afectadas por los eventos ligados a El Niño. De estas, se reportaron 31 fallecidas, 6 desaparecidas y 807 albergadas, a consecuencia principalmente de las inundaciones y deslaves. El balance de viviendas registró 4,475 afectadas, 184 destruidas y 153 escuelas dañadas.

En Perú, debido a los excesos en las precipitaciones, se identificaron 410 mil afectados, 16 mil damnificados, 63 heridos y 24 fallecidos. El número de viviendas afectadas fue de 44,399, siendo 2,127 destruidas. Se reportaron alrededor de 400 colegios afectados y 72 centros de salud dañados. En la agricultura, unas 695 mil hectáreas de cultivos fueron destrozados y más 152 mil hectáreas fueron afectadas, según el Consejo Nacional de Gestión de Riesgo del Fenómeno de El Niño [CONAGER-FEN] (ABC Internacional, 2016; El Comercio, 2016b). 
Las inundaciones en Bolivia dejaron 19 fallecidos, cerca de 60 mil familias se vieron afectadas, 31 mil hectáreas de cultivos y casi 16 mil cabezas de ganado se perdieron. Las regiones más impactadas fueron las del altiplano, en el sector occidental del país, según indicaron las autoridades de Defensa Civil del Gobierno Plurinacional de Bolivia (Ultima Hora, 2016).

En Chile, las inundaciones dejaron 31 personas fallecidas, 16 desaparecidos y 16,588 damnificados. Hubo también daños materiales, pérdidas de infraestructuras, viviendas e ingeniería civil (Oficina Nacional de Emergencia del Ministerio del Interior [ONEMI], 2015; Cooperativa, 2015).

En la región, el sector salud se vio afectado por el incremento de enfermedades como el cólera, la malaria, infecciones estomacales y conjuntivitis (Defensoría del Pueblo del Perú, 2015). En los países afectados por la sequía, tales como Colombia, Venezuela y algunas partes de Ecuador, se incrementaron los problemas alérgicos por la resequedad de la mucosa respiratoria, infecciones en la piel por la falta de agua para el aseo personal y enfermedades transmitidas por la mala cocción de los alimentos o su inadecuada limpieza (Telesur, 2016).

\section{Discusión}

El evento El Niño 2015-16 fue de los más vigilados por los centros globales y regionales. Con varios meses de anticipación fue posible comunicar al mundo lo que los científicos venían observando y la posible evolución del evento. Los medios de comunicación a nivel global informaron sobre lo que podría ocurrir dada la magnitud con que se esperaba que se desarrolle el evento y como de hecho sucedió.

A pesar de la magnitud de los impactos negativos observados, es necesario destacar el amplio avance conseguido en el conocimiento, seguimiento y monitoreo de El Niño, una vez que se tiene cierto grado de certeza de su ocurrencia antes de sus primeras manifestaciones. Para 2015-16 ya se contaba con una red informativa a nivel internacional, de organismos, agencias y centros que han facilitado informaciones de monitoreo y alertas para promover la concienciación, prevención y adaptación a todo nivel. Esto en algunos casos ayudó a reducir los impactos negativos, de eventos similares como en 1982-1983 y 19971998 (Banco de Desarrollo de América Latina [CAF], 2000).

La falta de cohesión institucional resulta en una inadecuada gestión en el territorio e ineficiente distribución de recursos. Para el caso de El Niño 2015-16, este pudo haber sido uno de los aspectos cruciales en algunos de los países.

Las inundaciones producen daños inmediatos a los cultivos y se ha prestado bastante atención a los impactos negativos de estas. Sin embargo la sequía que se ve exacerbada en algunos países por los eventos como El Niño 2015-16, no es tan evidente, lo que provoca que en algunos casos no se le presta la atención requerida.

El crecimiento demográfico y la limitada gestión del ordenamiento territorial han incrementado los riesgos climáticos. Este es el caso de las periferias urbanas en zonas inundables, la ocupación de cauces naturales, la falta de gestión de residuos y la despreocupación institucional en muchos casos de estas zonas habitadas de forma irregular. Esta problemática se identificó en El Niño 1997-1998 (CAF, 2000) y aún es un agravante que sigue manteniendo la alta vulnerabilidad del territorio.

\section{Conclusiones}

En algunos casos la génesis, desarrollo y magnitud son parecidos con otros eventos, no obstante, se debe considerar que, aunque los eventos son similares, estos pueden no tener las mismas características y afectaciones en las mismas regiones. Sin embargo, la mejor forma de enfrentar los impactos es todavía por la experiencia del pasado.

Para el monitoreo integral del evento El Niño, se debe promover el máximo uso de las fuentes de información calificadas del sistema de vigilancia climática mundial [OMM] que incluye a los Centros de Producción Global, los Centros Regionales y los Servicios Meteorológicos e Hidrológicos Nacionales [SMHN] en cada país.

Es necesario establecer a escala regional, un protocolo dentro del sistema de Naciones Unidas para la comunicación y coordinación relacionados con El Niño/La Niña con base en las fuentes antes mencionadas. 
Para promover los aspectos comunicacionales y la participación de los diversos sectores se debe fortalecer el funcionamiento rutinario de los Foros o mesas climáticas nacionales que incluyan a los representantes sectoriales y los de gestión de riesgos.

Es fundamental fortalecer las capacidades de los SMHN en cuanto a análisis climático, predicción estacional, el uso de las fuentes globales y regionales de información así como el manejo de estrategias de comunicación hacia sus autoridades.

Los SMHN deben apoyarse de la operación de los Centros Climáticos Regionales para la implementación de sistemas de información sobre vulnerabilidad e impactos, para lo cual estos centros deben también ser fortalecidos.

Es importante impulsar a nivel de las organizaciones internacionales y de ayuda humanitaria, la coordinación y cooperación en asuntos climáticos con énfasis en la sequía.

En algunos casos la influencia mediática de la prensa también interfiere en la correcta comprensión de los impactos y en la planificación para afrontar el evento climático. Por ello, los medios de comunicación, las agencias y organismos que brindan información deben ser conscientes de la importancia de la información que se proporciona. Para mejorar esta situación se necesita implementar en cada país, redes de voceros voluntarios del clima para cubrir la difusión hacia los usuarios finales.

La conectividad vial y la construcción de infraestructuras civiles todavía desconocen en buena parte, los períodos de recurrencia de los eventos climáticos extremos. Las normas y códigos de construcción deben ponerse en vigor.

Se debe capitalizar al máximo la presencia de los organismos gubernamentales regionales (Unión de Naciones Suramericanas [UNASUR] o SICA son buenos ejemplos) para asegurar la coordinación entre países, asistencia mutua y el óptimo uso de los recursos de información.

Los indicadores de gestión territorial son imprescindibles para dar correcto seguimiento a la integración de estas prácticas en las políticas de desarrollo, de manera que se pueda analizar periódicamente y corregir.

El fortalecimiento institucional debe ir orientado, a la cooperación interinstitucional. En segundo lugar, debe forjarse una proyección de la planificación adaptativa a largo plazo, tomando como base las experiencias logradas en los eventos climáticos pasados. Una sociedad preparada para afrontar la variabilidad del clima está mejor preparada para afrontar los efectos del cambio climático. Es necesario armonizar las agendas de Gestión de Riesgos y Cambio Climático, pues la gestión de riesgo al clima presente es el primer paso para la adaptación factible.

\section{Referencias}

ABC Internacional (2016). El fenómeno climático "El Niño" azota gran parte de Perú. Recuperado de http:// www.abc.es/internacional/abci-fenomeno-climatico-nino-azota-4780026725001-20160229093500 video.html

Bureau of Meteorology [BOM] Australia (1997). Recuperado de http://www.bom.gov.au/cgi-bin/ oceanography/wrap_ocean_analysis.pl?id=IDYOC007\&year=1997\&month=01

Bureau of Meteorology [BOM] Australia (2015). Recuperado de http://www.bom.gov.au/cgi-bin/ oceanography/wrap_ocean_analysis.pl?id=IDYOC007\&year $=2015 \&$ month $=01$

Banco de Desarrollo de América Latina [CAF] (2000). El Fenómeno El Niño 1997 - 199.8 Memoria, Retos y Soluciones. Volumen IV Ecuador. Sección 4. Corporación Andina de Fomento - [CAF].

Center, C. P. (2009). El Niño/Southern Oscillation (ENSO) Diagnostic Discussion. Camp Springs, Maryland: NOAA/National Weather Service.

Comisión Económica para América Latina y el Caribe [CEPAL] (2011). The Economics of the Climate Change in the Caribbean. Caribbean Development Report, Naciones Unidas - Comisión Económica para América Latina y El Caribe [CEPAL]. Volume III. pp. 150. Recuperado de http://www.cepal.org/ publicaciones/xml/2/45412/LCARL.346.pdf 
Cooperativa (2015). Las consecuencias de la lluvia en la zona centro norte. Recuperado de http:// www.cooperativa.cl/noticias/pais/tiempo/las-consecuencias-de-la-lluvia-en-la-zona-centronorte/2015-03-24/074058.html

Defensoría del Pueblo del Perú (2015). Conoce las consecuencias del fenómeno El Niño y la responsabilidad que tienen las autoridades para afrontar los embates de la naturaleza. Recuperado de http://www. defensoria.gob.pe/blog/conoce-las-consecuencias-del-fenomeno-el-nino-y-la-responsabilidad-quetienen-las-autoridades-para-afrontar-los-embates-de-la-naturalezal

El Comercio (2016a). Perú prepara su actividad pesquera para enfrentar el cambio climático. Recuperado de http://www.elcomercio.com/tendencias/peru-cambioclimatico-piura-pesca-fenomenodeelnino.html

El Comercio (2016b). Casi 9 mil damnificados deja fenómeno de El Niño hasta ahora. Recuperado de http://elcomercio.pe/sociedad/peru/casi-9-mil-damnificados-deja-fenomeno-nino-hasta-ahoranoticia-1890000

El Universo (2016). El Niño reduce volumen de pesca en el Pacífico y Caribe centroamericano. Recuperado de http://www.eluniverso.com/noticias/2016/03/16/nota/5468961/nino-reduce-volumen-pesca-pacificocaribe-centroamericano

Fan, Y. \& Van den Dool, H. (2008). A global monthly land surface air temperature analysis for 1948-present. Journal of Geophysical Research: Atmospheres, 113(D1). http://dx.doi.org/10.1029/2007JD008470

Food and Agriculture Organization of the United Nations [FAO] (2015). El Niño provoca cuantiosas pérdidas en las cosechas de América Central. Recuperado de http://www.fao.org/news/story/es/ item/328686/icode/

Food and Agriculture Organization of the United Nations [FAO] (2016a). El Niño and La Niña: Preparedness and Response. 5 pp. Recuperado de http://www.fao.org/fileadmin/user_upload/emergencies/docs/ FAOElNinoSitRep_versionJULY.pdf

Food and Agriculture Organization of the United Nations [FAO] (2016b). 2015-2016 El Niño. Early action and response for agriculture, food security and nutrition. pp 44. Recuperado de http://www.fao. org/3/a-i5855e.pdf

Food and Agriculture Organization of the United Nations [FAO] (2016c). GIEWS Country Brief - Brazil. Global Information and Early Warning System on Food and Agriculture [GIEWS]. 2 pp. Recuperado de http://reliefweb.int/sites/reliefweb.int/files/resources/BRA_8.pdf

Food and Agriculture Organization of the United Nations [FAO] (2016d). Dry Corridor Central America. Situation Report - June 2016. 3 pp. Recuperado de http://www.fao.org/fileadmin/user_upload/ emergencies/docs/DryCorridor-SitRepJune2016.pdf

Gestión (2015). APESEG: Impacto del Fenómeno de El Niño generaría pérdidas de hasta US\$3,700 millones. Recuperado de http://gestion.pe/economia/apeseg-impacto-fenomeno-nino-generaria-perdidashasta-us-3700-milllones-2140897

Janowiak, J. E., \& Xie, P. (1999). CAMS-OPI: A global satellite-rain gauge merged product for real-time precipitation monitoring applications. Journal of Climate, 12(11), 3335-3342.

Oficina Nacional de Emergencia del Ministerio del Interior [ONEMI] (2015). Monitoreo por evento hidrometeorológico 07/06/2015. Oficina Nacional de Emergencia del Ministerio del Interior y Seguridad Pública [ONEMI]. Recuperado de http://www.onemi.cl/alerta/monitoreo-por-eventohidrometeorologico/

Organización Meteorológica Mundial [OMM]. (2016). Boletín El Niño/La Niña Hoy. 18 febrero 2016. Organización Meteorológica Mundial [OMM]. Recuperado de http://www.wmo.int/pages/prog/wcp/ wcasp/documents/WMO_ENSO_Feb16_Esp.pdf

Programa Mundial de Alimentos [WFP] (2016a). El Niño 2015-2016, Preparedness and Response. Situation Report \# 3. World Food Programme [WFP]. 8 pp. Recuperado de http://documents.wfp. org/stellent/groups/public/documents/ep/wfp282093.pdf 
Programa Mundial de Alimentos [WFP] (2016b). Haiti Emergency Food Security Assessment. World Food Programme [WFP]. pp 4. Recuperado de http://reliefweb.int/sites/reliefweb.int/files/resources/ Haiti\%20Emergency\%20Food\%20Security\%20Assessment\%20February\%202016.pdf

RCN Radio (2016). Fenómeno del Niño afectó 600 mil hectáreas de café en Colombia. Recuperado de http:// www.rcnradio.com/locales/fenomeno-del-nino-afecto-600-mil-hectareas-cafe-colombia/

Reynolds, R. W., Rayner, N. A., Smith, T. M., Stokes, D. C., \& Wang, W. (2002). An improved in situ and satellite SST analysis for climate. Journal of climate, 15(13), 1609-1625. https://doi.org/10.1175/15200442(2002)015<1609:AIISAS>2.0.CO;2

Secretaria de Gestión de Riesgos [SGR] (2016). Informe Situación Actual No 95. Recuperado de http:// enosecuador.com/informes/category/31-informes-de-situacion

Telesur (2016). Conozca los efectos de El Niño en América Latina. Recuperado de http://www.telesurtv.net/ news/Conozca-los-efectos-de-El-Nino-en-America-Latina-20160112-0044.html

Ultima hora (2016). Bolivia reporta 19 muertos por efectos del fenómeno El Niño desde noviembre. Recuperado de http://www.ultimahora.com/bolivia-reporta-19-muertes-efectos-del-fenomeno-elnino-noviembre-n974983.html

United Nations Office for Disaster Risk Reduction [UNISDR]. (2016). The human cost of the hottest year on record. United Nations Office for Disaster Risk Reduction [UNISDR]. Recuperado de http://www. unisdr.org/archive/47791

United Nations Office for the Coordination of Humanitarian Affairs [OCHA]. (2015). Crisis Humanitaria Sequía 2015. Reporte de Situación No. 3. United Nations Office for the Coordination of Humanitarian Affairs [OCHA]. Recuperado de http://www.redhum.org/uploads/documentos/pdf/Redhum_GT_ Informe de Situacion_No_3_Inseguridad_Alimentaria_Nutricional_sequia_EHP_20150900120150901-IC-16971.pdf

United Nations Office for the Coordination of Humanitarian Affairs [OCHA]. (2016). El Niño: Overview of impact, projected humanitarian need and response, 21 September 2016. United Nations Office for the Coordination of Humanitarian Affairs [OCHA]. Recuperado de http://reliefweb.int/report/world/elni-o-overview-impact-projected-humanitarian-needs-and-response-21-september-2016

Univisión (2015). Huracán Patricia Golpea También Economía de México. Recuperado de http://www. univision.com/noticias/huracan-patricia/huracan-patricia-golpea-tambien-economia-de-mexico 